\title{
REMOTE COLLABORATIVE DATA MINING THROUGH ONLINE KNOWLEDGE SHARING
}

\author{
Alípio Jorge ${ }^{1}$, Steve Moyle ${ }^{2}$, Angi Voß ${ }^{3}$ \\ 'LIACC-FEP, University of Porto, PORTUGAL amiorge@liacc.up.pt, \\ ${ }^{2}$ Oxford University Computing Laboratory UK, sam@comlab.ox.ac.uk \\ ${ }^{3}$ Fraunhofer-Gesellschaft zur Foerderung, Sankt Augustin, GERMANY. \\ angi.voss@ais.fraunhofer.de
}

\begin{abstract}
The basic principles of a methodology for remote collaborative data mining are proposed. Starting from CRISP-DM, a general data mining process designed to carry out data mining projects; it is described how the principles of knowledge sharing and ease of communication can be embedded in the data mining process. The aim is to allow the execution of data mining projects, with the participation of multiple experts working from distant locations. All the participants in such a project can profit from the knowledge produced by others and share their knowledge online with the other participants. The produced knowledge (for example data transformations, working hypothesis, models, results of experiments) is also stored for future inspection and use, in pursuit of organizational learning. A prototypical implementation (RAMSYS) of the remote collaborative methodology is described with examples.
\end{abstract}

\section{INTRODUCTION}

In many technical and scientific domains, expertise is spread throughout a number of different locations. In such a setting, to solve a specific problem implies that either experts must meet at one location, with high costs, or solving the problem with local available expertise, which often leads to a sub-optimal solution. An intermediate possibility is remote collaboration, where each expert or team of experts located at a specific geographic point communicate with the other teams whenever necessary. The following issues should be considered when developing a remote collaborative problem-solving framework:

a Problem solving methodology: A specific problem solving methodology must be known and utilized by all the participants. One way to ensure this is to embed the problem solving methodology in a remote collaboration tool that is used for both problem solving and communication.

- Knowledge sharing: Knowledge should be shared for both the immediate problem solving and for the greater organizational good. When some piece of knowledge relevant to the project is produced by one of the experts it should be made available as soon as possible to the other experts. Furthermore, knowledge produced and retained enables organizational learning (Voß et al. 2001). 
Dase of communication: The problem solving itself should take most of the participant's time. Communication between experts should be made easy and enjoyable or even transparent. Tiwana (Tiwana 2000) suggests that using such a system should be as natural as using the telephone.

One important case is Data Mining. The leading edge of European Data Mining expertise is spread over a few research groups and companies across Europe. Each one of these units has specific expertise to solve particular data mining problems, which motivates collaborative work. In this paper we describe a collaborative data mining methodology, its principles and a system that implements it.

\section{DATA MINING PROBLEM SOLVING METHODOLOGY}

Data Mining can be loosely described as the process of automatically extracting useful knowledge from large collections of data (Adriaans and Zantige, 1996; Fayyad et al. 1996). In this context, data can come from many different domains, such as a marketing relational database, medical records, DNA, plain text or the access logs of a web site. The extracted knowledge is typically represented as logical and/or mathematical models.

The various sorts of data mining problems that arise allow different approaches and tools. Given a data mining problem, one important challenge is to find the most appropriate team of available experts. One of the aims of the IST Project Sol-Eu-Net (Lavrac et al. 2002) is to develop a dynamic network of expert teams with extensive experience in data mining and decision support with complementary skills oriented towards solving practical knowledge discovery problems.

To approach Data Mining problems, the Sol-Eu-Net Project has adopted the CRISP-DM methodology - CRoss Industry Standard Process for Data Mining (Chapman et al.2000). In CRISP-DM, six interrelated phases are used to describe the data mining process: business understanding, data understanding, data preparation, modelling, evaluation, and deployment. Figure 1 presents an overview of the CRISP-DM reference model. The phases that can most easily be performed in a remote-collaborative fashion are Data preparation, and Modelling.

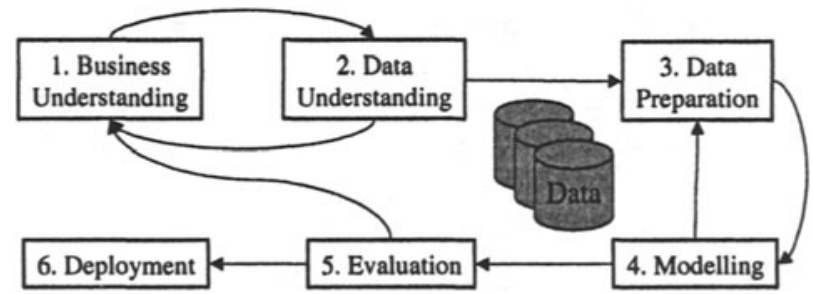

Figure 1 - The CRISP Data Mining Process (Chapman et. al. 2000).

Modelling is the process of extracting input/output patterns from data. The derived models - typically mathematical or logical models - allow such patterns to be applied to unseen data. Modelling necessarily relies heavily on the preceding data preparation phase. This data preparation includes the selection of tables, records and attributes as well as data transformations and cleaning. In the modelling phase, 
various techniques are applied and for each of them their parameters are calibrated to optimal values. The resulting models are then tested and interpreted in the evaluation phase.

\section{KNOWLEDGE SHARING}

The use of a common methodology enhances communication and knowledge sharing between participants of a collaborative data mining project. This can be done at two different levels: First, during each project, knowledge can be shared online to boost results; second, after the end of the project, knowledge related to the problem solving procedure can be analyzed as part of the organizational learning process.

Table 1- Data Mining Problem Knowledge.

- Problem Definition

- The business understanding and the data mining view of the problem.

- Distilled Knowledge from Related problems

- Similar problems addressed in the past, applied solutions, literature and produced knowledge.

- Evaluation Criteria Definition

- How is the solution to be evaluated?

- Data

- The collected database $\left(\mathrm{DB}_{0}\right)$, Meta-data, Quality of data (e.g. missing values, inconsistent records), Derived data and information on how to repeat the derivation process from $\mathrm{DB}_{0}$.

- Idea Board

- $\quad$ Problem specific knowledge derived during problem solving.

In remote collaborative work, team members communicate with each other through telecommunication media. These exchanges are automatically recorded in a way that makes it easy for new experteise to join the team at any time, or for other members to resume work after shorter or longer individual absences. Therefore these tools and methods for distributed collaboration provide a basis for capturing knowledge (from experiences) and, vice versa, and also that the tools and methods for applying knowledge help to improve collaboration.

In the methodology utilized here, the knowledge to be shared corresponds to the current best understanding of the problem. This consists of the problem definition, the data, hypotheses (ideas) about the data and the models, and the current validity status of any hypotheses. This knowledge is sketched in Table 1.

\section{COLLABORATIVE DATA MINING}

One of the aims of the Sol-Eu-Net Project (Lavrac et al., 2002) is the development of a virtual enterprise that produces data mining and decision support services to enhance European business competitiveness. Customers of Sol-Eu-Net are able to pose their data mining problem to a multitude of experts from academic and commercial partners, located in six European countries. The experts apply their methods to solving the problem - but also communicate with each other to share 
their growing understanding of the problem. It is here that collaboration is key. Other important matters such as legal issues, intellectual property rights, contracting and payment are extremely important in an enterprise context,, but these issues will not be addressed here.

The process of analysing data through models has many similarities to experimental research: first the modeller (cf. a scientist) applies his/her analysis techniques to the data (cf. experimental results) to produce a model (cf. a new theory). These models (cf. theories) are tested for significance, and then refined (maybe in the light of new evidence). Like the process of scientific discovery, data mining can benefit from different techniques used by multiple researchers who collaborate to improve their combined understanding. This collaborative approach is what Sol-Eu-Net aims to provide. According to (Moyle et al., 2000) collaboration may be based on the following principles.

- Light Management: Project objectives, requirements, problem definition, and evaluation criteria should be clear from the beginning to all participants. Consequently, each project should have a management structure that ensures that information flows within the network and that a good solution is provided. However, management should not control directly the work of each unit.

- Start at any time: Extra expertise may often be necessary in solving a data mining problem. Thus, all the information necessary to start problem solving should always be available. This includes problem definition, data, evaluation criteria and any specific knowledge already produced by project participants. The collaborative methodology satisfies the start any time requirement if any competent new participant can enter the project in a minimal amount of time without having to communicate directly with any other participant.

- Stop at any time: Problem solving should be conducted by each unit so that a working solution (model) is available whenever the management committee requests a result. One approach would be that participants follow a sort of "simplicity first" strategy for modelling: simpler models are tried first, and more complex models are compared to the simpler ones.

口 Problem Solving Freedom: Members of the network have varied expertise, techniques, and tools. It is hoped that these complement each other in the data mining process. Each unit is in the best position to decide which approach to follow for the given problem. The management structure may give specific suggestions but does not prescribe problem-solving approaches. In this way, experts are not constrained as to which techniques to employ.

- Online Knowledge Sharing: Any new knowledge on the problem produced by participants should be immediately shared within the data mining project (e.g. ideas, data modifications, models, evaluation results).

a Security: The data and information relating to any data mining problem is likely to contain sensitive information, which may not be revealed outside the project. Access to project information should be strictly controlled.

These principles of collaboration and the CRISP-DM methodology as adapted for the Sol-Eu-Net Project lead to the requirements for a support system called RAMSYS, a "SYStem for RApid remote collaboration in data Mining projects". 


\section{THE RAMSYS SYSTEM}

Through its tasks, the CRISP-DM methodology provides a framework for organising the work of the units and the deliverables. Every task can be assigned to one or more units. The deliverables produced by each unit are associated with their individual assignment. The structure of tasks, assignments and deliverables records who did (or has to do) what in a project, is an important instrument for achieving the principle of "light management".

The principles of "problem solving freedom" and "online knowledge sharing" require a combination of tools that allow the groups to share data, data transformations and metadata, to apply models to training data and to assess or evaluate models using evaluation data. This is called the "data mining laboratory". The principles "start / stop at any time" call for a means that, at a glance, provides an overview of what is the current state of the problem (i.e. was done, how and why). This is the current version of the problem information (see Table 1). On an idea board the rationale (justifications, the why) for the current solution strategy are recorded. Here the units exchange ideas (hypotheses) about expected outcomes, promising models or alternative parameter settings. They can also discuss and explain successes and failures. An experimentation area visualises the history and current status of the problem. It allows the units to record what actions should be performed, are being performed or have been performed on which data. The documentation of such experiments is a natural component of any data minining consultants work. The experimentation area is operationally coupled with the data mining laboratory. It provides an intuitive, convenient access to the tools and results in the data mining laboratory.

- Idea board On the idea board the users (here a "user" is typically a data mining expert assigned to a particular data mining problem) share ideas about which final results (patterns, relationships, insights) to expect, which models to apply, or which transformations to execute on the data.

a Data mining laboratory the laboratory includes a data warehouse as well as the modeling ones. These allow data transformations to be performed in and shared via secure-encrypted access channels. Each transformation is linked to its rationale in the idea board. The data transformations are presented as a sequence of operations starting from the original dataset. To facilitate "blind" assessment of any submitted models, the "outcome" attributes of some records (called the "test set") will not be accessible to the data miners until the evaluation phase of the data mining project.

- Experimentation area The experimentation area contains two kinds of objects: objects that represent actions, and objects that represent their input and output. These are called action objects and data objects. Action objects are transformations, model applications, and assessments of models (i.e. applications of models to test data). Data objects may be views, metadata, and results of model applications and assessments. Data objects and action objects are interrelated with many input/output relationships

a Task management The six phases of the CRISP-DM methodology and their sub-tasks (generic tasks and specialised tasks) form a hierarchy. In the course of a data mining project, each task gets associated with one or more assignments. An assignment of a task can also specify the unit, the budget, the deadline for 
example. Assignments are related to ideas that motivate, justify or describe the approach chosen in the assignment.

a The portal All services are integrated into a Web portal. Naturally, secure access applies to all relevent information. For each data mining project thereis a summary of recent changes different views into the task, experiment, idea and parts of the project.

\section{IMPLEMENTATION}

The SoEuNet Project is realising the RAMSYS system with open source software Zeno, which is a web-based groupware system (Gordon et al. 2001). The firsst prototype of RAMSYS was limited, using a customised system of folder-like structures, discussion forums and an address book. The key conceptual components of the system are:

The journal server: Application-specific data structures, such as data objects and action objects, can be mapped onto two Zeno-specific concepts, journals and articles. Journals and articles resemble folders in their ability to contain other information resources, but differ from them due to their special functionality in the context of Zeno.

- User interface: Zeno comes with generic user interfaces. With the Web interface users can navigate through the hierarchy of journals. A custom interface for RAMSYS is under development and will be available in early 2002.

These facilities, combined with disciplined application of the methodology, allowed the crude prototype to be tested. For example data mining participants were able log on through an ordinary browser. The structure of folders mainly followed the CRISP-DM phases, tasks and outputs (such as reports). The folders also contained datasets (or links to datasets), data transformations, metadata, produced models and results of evaluating those models. Besides this information, there was a structured discussion forum (akin to a newsgroup) that is accessed through Zeno in the same session. The discussion forums emulated the idea board described above. A fully customized version of the Zeno system to support RAMSYS will be available for use in early 2002 .

\section{THE METHODOLOGY IN ACTION}

The collaborative data mining methodology is now being used in two real life data mining problems, and has been tested before on one challenge problem. Currently, the methodology is restricted by the fact that the communication tools are not yet developed to the desired level. Nevertheless, just by following the basic principles of the methodology, a large structured knowledge repository for each of the projects is being gathered. This enables a smoother entrance of newcomers to the project (startat-any-time principle) and helps retain the organizational memory.

Even with this very limited experience in using the prototype of a remote collaborative data mining framework some very important results have been 
achieved. First, the need for collaboration motivates the use of a well-defined methodology, which is being followed by the participants in both projects. Second, by following the methodology, a large base of shareable knowledge about the data mining problem, containing for example business understanding reports, problem specification, data understanding and data preparation reports, grows very quickly, and can be used by current and future participants. This knowledge about the problem is also a good source for extracting lessons learned when the problem terminates - this is an instance of the generation of re-usable knowledge.

In one data mining problem (related to direct marketing), this methodology was utilized. In studying the problem solving history and collaborative workflow, clear evidence was produced that beneficial remote collaboration had occurred. For example one member of one of the data mining teams performed some data preprocessing to produce a new data set. These transformations were then used by five other sets of data mining experts as input to their modeling processes.

One positive observation is that, although participants like the idea of sharing their knowledge, they are reluctant to describe every step in their problem solving procedure until they actually reach some result that is considered as "good". This is mainly due, in our opinion, to the current lack of integration between data preparation/modeling and communication in the current version of the collaborative data mining support tool.

\section{RELATED WORK}

In the fields of data mining and software development, some other projects are relevant to the collaborative methodology that is reported here. In the Metal Esprit Project (Brazdil and Soares, 2000), the aim is to collect information about datasets and the performance of different pre-processing and modeling algorithms on those datasets to guide a "lone" data miner in the modeling phase. The aid consists in recommending specific algorithms for specific datasets, reducing experimentation time. The Project MiningMarts (Kietz et al., 2000) also aims at collecting information about data mining problems and solutions to help solving new data mining problems. Adaptation of solutions is done through case based reasoning.

In the software development arena, many open source development platforms have appeared recently with the advent of the Internet. One important example is the SourceForge platform, where any open source software development project can be started, open to remote collaboration, maintaining a project schedule, a bug fix log and a control over versions.

\section{CONCLUSION AND FUTURE WORK}

Remote collaborative problem solving is a major requirement for virtual enterprises, by enabling the exploitation of available complementary skills located at different sites, and also to retain the organizational memory about what has been done in each problem solving instance. 
Data mining is one such problem domain. In the Sol-Eu-Net Project the aim is to establish a team of experts to handle a given data mining (or decision support) problem. The collaborative methodology proposed largely follows CRISP-DM, but takes into account the specific needs of online knowledge sharing. A first prototype of RAMSYS, the tool that implements the methodology, is operational and has been used on more than one data mining project. Although we regard the early results as reassuring, it is evident that a more transparent knowledge sharing protocol, highly integrated with the problem solving tools is required. This way, each participant could concentrate on the problem solving, leaving the communication and the online knowledge sharing concern to the system. On the other hand, each participant could, at any time, see the knowledge produced by others. Furthermore, (s)he could be automatically notified anytime something relevant would appear on the idea board (such as the production of a model on a data transformation proposed by him/her).

\section{ACKNOWLEDGEMENTS}

The work reported in this paper was supported by the IST-1999-11495 project SolEu-Net, Data Mining and Decision Support for Business Competitiveness: A European Virtual Enterprise. The first author also thanks the Portuguese Programa de Financiamento Plurianual de Unidades de I\&D (FCT). The authors are grateful for the help of Gernot Richter in the preparation of this paper.

\section{REFERENCES}

Adriaans, P, and Zantinge, D., Data Mining, Addison-Wesley, 1996.

Brazdil, P. and Soares, C., A comparison of ranking methods for classification algorithm selection, in Machine Learning, Proceedings of the ECML'OO - $11^{\text {th }}$ European Conference on Machine Learning, Ed. R. L. Mántaras and E. Plaza. Barcelona, 2000.

Chapman, P., J. Clinton, R. Kerber, T. Khabaza, T. Reinartz, C. Shearer, and R Wirth, CRISP-DM 1.0: Step-by-step data mining guide. CRISP-DM consortium, 2000.

Fayyad, U., Piatetsky-Shapiro, G., Smyth, P., and Ramasamy, U. (editors), Advances in Knowledge Discovery and Data Mining, MIT Press, 1996.

Gordon, T.F., A. VoB, G. Richter, and O. Marker, Zeno: Groupware for Discourses on the Internet, KI Kunstliche Intelligenz, Vol. 15, pp. 43-45, 2001.

Kietz, J.-U., R. Zücker, and A. Vaduva: Mining Mart: Combining Case-Based-Reasoning and MultiStrategy Learning into a Framework for Reusing KDD-Applications, In: Proc. of the 5th Int. Conf. on Multi-Strategy Learning, Guimarães, Editors Brazdil, P. and Michalski R., University of Porto, 2000.

Lavrac, N., Urbancic, T., Orel, A. Virtual Enterprise For Data Mining And Decision Support: A Model For Networking Academia And Business, submitted to PROVE-2002.

Moyle, S., A. Jorge, and C. Leandro: RACODAMISYS - a methodology and tool for supporting rapid remote collaborative data mining projects, LIACC Technical Report, University of Porto, 2000.

Ruhe, G. and F. Bomarius (eds.): Learning Software Organization - Methodology and Applications. Springer-Verlag. Lecture Notes in Computer Science, Volume 1756, 2000.

Tiwana, A. The Knowledge Management Toolkit: Practical techniques for building a knowledge management system. Prentice Hall, New Jersey, 2000.

VoB, A., Richter, G., Moyle, S., Jorge, A, Collaboration support for virtual data mining enterprises, K.-D. Althoff, R.L. Feldmaann and W. Mullen (Eds), Learning Software Organizations - LSO 2001, LNCS 2176, Springer Verlag, pp 83-95, 2001. 